\title{
Terapia medicamentosa segura: perspectivas da enfermagem e da farmácia no cuidado de paciente em Unidade de Terapia Intensiva (UTI)
}

Safe medicinal therapy: perspectives of nursing and pharmacy in patient care in the Intensive Care Unit (ICU)

Terapia medicinal segura: perspectivas de enfermería y farmacia en la atención al paciente en la Unidad de Cuidados Intensivos (UCI)

\section{Resumo}

Diante do número alarmante de eventos adversos relacionados a medicamentos no âmbito da Unidade de Terapia Intensiva (UTI), ações efetivas no cuidado do paciente são necessárias. Nessa perspectiva, este trabalho tem como objetivo discutir o papel da enfermagem e da farmácia quanto à utilização segura de medicamentos, visando garantir a qualidade da assistência prestada a pacientes críticos. Foi realizada uma revisão integrativa, de caráter exploratóriodescritivo com abordagem qualitativa, na qual resultou na seleção de 21 artigos, publicados entre janeiro e abril de 2021, indexados nas principais bases de dados. A partir dos estudos analisados, observou-se que os principais eventos adversos estavam relacionados a erros de prescrição, preparo e administração; ocorrência de interações medicamentosas; incompatibilidades entre drogas e equipamentos. $\mathrm{O}$ surgimento desses problemas foi associado à falha de comunicação da equipe multiprofissional, a sobrecarga de trabalho e a falta de conhecimento sobre farmacodinâmica e farmacocinética da droga. Portanto, faz-se necessário a integração e articulação de enfermeiros e farmacêuticos para que a farmacoterapia seja efetiva, segura e assertiva, mediante a complexidade projetada na prática, demandando desses profissionais maior capacitação e treinamento.

Palavras-chave: Cuidados de enfermagem; Serviço de farmácia clínica; Unidades de terapia intensiva; Erros de medicação; Segurança do paciente.

\begin{abstract}
In view of the alarming number of adverse events related to medications within the scope of the Intensive Care Unit (ICU), effective actions in patient care are necessary. In this perspective, this work aims to discuss the role of nursing and pharmacy regarding the safe use of medicines, aiming to guarantee the quality of care provided to critically ill patients. An integrative review of an exploratory-descriptive character with a qualitative approach was carried out, which resulted in the selection of 21 articles, published between January and April 2021, indexed in the main databases. From the studies analyzed, it was observed that the main adverse events were related to errors in prescription, preparation and administration; occurrence of drug interactions; incompatibilities between drugs and equipment. The emergence of these problems was associated with the communication failure of the multidisciplinary team, the work overload and the lack of knowledge about the pharmacodynamics and pharmacokinetics of the drug. Therefore, it is necessary to integrate and articulate nurses and pharmacists so that pharmacotherapy is effective, safe and assertive, due to the complexity projected in practice, demanding from these professionals greater qualification and training.
\end{abstract}

Keywords: Nursing care; Clinical pharmacy service; Intensive care units; Medication errors; Patient safety. 


\begin{abstract}
Resumen
Ante la alarmante cantidad de eventos adversos relacionados con los medicamentos en el ámbito de la Unidad de Cuidados Intensivos (UCI), son necesarias acciones efectivas en la atención al paciente. En esta perspectiva, este trabajo tiene como objetivo discutir el papel de la enfermería y la farmacia en el uso seguro de los medicamentos, con el objetivo de garantizar la calidad de la atención brindada a los pacientes críticos. Se realizó una revisión integradora de carácter exploratorio-descriptivo con enfoque cualitativo, que resultó en la selección de 21 artículos, publicados entre enero y abril de 2021, indexados en las principales bases de datos. De los estudios analizados se observó que los principales eventos adversos estuvieron relacionados con errores en la prescripción, preparación y administración; aparición de interacciones farmacológicas; incompatibilidades entre medicamentos y equipos. La aparición de estos problemas se asoció con la falla de comunicación del equipo multidisciplinar, la sobrecarga de trabajo y el desconocimiento de la farmacodinámica y farmacocinética del fármaco. Por tanto, es necesario integrar y articular enfermeros y farmacéuticos para que la farmacoterapia sea eficaz, segura y asertiva, debido a la complejidad proyectada en la práctica, exigiendo a estos profesionales una mayor cualificación y formación.
\end{abstract}

Palabras clave: Atención de enfermería; Servicio de farmacia clínica; Unidades de cuidados intensivos; Errores de medicación; Seguridad del paciente.

\title{
1. Introdução
}

Os eventos adversos são considerados qualquer ocorrência médica desagradável manifestada durante o tratamento com um produto farmacêutico, mesmo que não haja necessariamente uma relação causal com este tratamento. Dessa forma, o estudo da temática sobre terapia medicamentosa em Unidade Terapia Intensiva (UTI) não pode ser dissociável à segurança do paciente, uma vez que esse faz uso de inúmeros medicamentos de diferentes classes e também devido ao seu estado clínico e hemodinâmico, o que pode levar a erros de medicação (Chapuis et al., 2019).

Segundo Farzi et al., (2017) os erros de medicação refere-se a qualquer evento evitável em cada estágio do processo de farmacoterapia, como prescrição, transcrição, distribuição de medicamentos e administração. Portanto, é possível compreender a importância do trabalho colaborativo da equipe multiprofissional para assegurar a qualidade assistencial. Esses erros colaboram para o aumento do tempo de hospitalização, incapacidade e morte em até 6,5\% das admissões hospitalares. Ao passo que, em média, os pacientes em UTI são expostos a 1,7\% erros/dia no qual 78\% dessas falhas estão relacionados a erros de medicação. Reis et al., (2017) relatam ainda, que 60\% dos erros estão relacionados à técnica do preparo e $75 \%$ à técnica de administração dos medicamentos intravenosos, tornando os dados extremamente alarmantes.

Nesta conjuntura, o profissional de enfermagem, além da função principal que é "o cuidar", ainda assume um papel fundamental durante o processo de utilização de medicamentos que engloba desde o recebimento e o preparo até a administração. Haja vista que, é possível a detecção precoce de falhas produzidas nas fases anteriores como: prescrição e dispensação (Guzzo et al., 2018). Assim, o enfermeiro consegue atuar como barreira para os possíveis eventos adversos referente a esse processo, sendo indispensável ter conhecimento quanto a farmacodinâmica e farmacocinética dos medicamentos utilizados, além da necessidade de articulação entre o serviço assistencial e o de farmácia para proporcionar maior segurança no preparo, na administração dos medicamentos e nos resultados esperados com menor probabilidade de erro possível (Borges et al., 2017).

Do mesmo modo, o farmacêutico clínico torna-se um profissional de grande valia para promoção da segurança do paciente, pois ele auxilia no uso racional de medicamentos, garantindo ao paciente uma farmacoterapia adequada, com resultados terapêuticos definidos, buscando sempre prevenir ou resolver os problemas relacionados a medicamentos (PRMs) de maneira comprometida, sistematizada e documentada (Gomes \& Reis, 2003).

Ademais, percebe-se ainda que se faz necessário ações efetivas de segurança do paciente nos serviços de saúde, com foco em executar diariamente tais ações, até que se tornem internalizadas pela equipe multiprofissional e façam parte da rotina do cuidado diário. Sendo assim, este trabalho objetiva discutir o papel da enfermagem e da farmácia quanto à utilização segura de medicamentos, visando garantir a qualidade da assistência prestada a pacientes internados em UTI. 


\section{Metodologia}

Trata-se de uma revisão integrativa, de caráter exploratório-descritivo com abordagem qualitativa na qual foram selecionados trabalhos, publicados entre janeiro e abril de 2021, indexados nas principais bases de dados: Scientific Eletronic Library Online (SciELO), Literatura Latino-americana e do Caribe em Ciências da Saúde (LILACS), PUBMED e Biblioteca Virtual de Saúde (BVS) (Pereira et al., 2018).

A pesquisa foi baseada na utilização dos descritores: Cuidados de Enfermagem; Serviço de Farmácia Clínica; Unidades de Terapia Intensiva; Erros de Medicação e Segurança do Paciente, a qual resultou na identificação de 44 artigos, sendo eleitos 21 artigos de relevância para desenvolvimento do tema. Foi considerado como critério de inclusão os artigos publicados nas línguas: inglês, português e espanhol e excluídas as literaturas com mais de 10 (dez) anos de publicação, dissertações e resumos publicados em anais de eventos. Os materiais foram analisados e selecionados com base na leitura dos títulos e posteriormente dos resumos. É válido ressaltar que também foram selecionados livros, protocolos, manuais e portarias do Ministério da Saúde que corroboram com a discussão do tema.

\section{Resultados}

\subsection{O cuidado de enfermagem na assistência a pacientes críticos}

Acredita-se que o cuidado a pacientes críticos precisa ser sistematizado e realizado de forma holística, pois dessa maneira aumenta a qualidade da assistência. O processo do cuidar não pode ser embasado, apenas, em sinais e sintomas e diagnóstico da patologia, mas sim centrado no paciente, em sua integralidade, nas condições de vida e nos determinantes e condicionantes sociais que o circundam (Arboit et al., 2020).

Hodiernamente o cuidado de enfermagem, nos serviços de UTI, vem sofrendo um declínio na qualidade, pois existem inúmeros fatores que levam a ocorrência de eventos adversos, como por exemplo a quantidade de medicamentos ministrados e suas vias de administração, os esquemas terapêuticos complexos e a gravidade do estado clínico (Mazzola et al., 2011). Segundo Cedraz e Junior (2014) a quantidade de medicamentos por prescrição, chegando até 15 medicamentos por paciente, é um indicador de risco para a ocorrência de IM e isso pode impactar diretamente no tempo e custo de internamento.

Um estudo transversal, realizado em um hospital filantrópico de Minas Gerais durante seis meses, analisou 309 prontuários de pacientes internados em UTI adulto. Foi encontrada a mediana de 7 medicamentos/paciente e a prevalência de exposição a interações medicamentosas contraindicadas (uso concomitante dos medicamentos não recomendada) ou maiores (interação que apresenta risco de vida ou eventos adversos sérios) foi de $81,8 \%$ ( $\mathrm{n}=251)$, ao passo que mais de um terço desses pacientes $(39,8 \%)$ foram expostos a quatro ou mais dessas IM simultaneamente. Entre as classes de medicamentos que estavam associadas ao elevado risco de IM estão: antieméticos, antiagregantes plaquetários, antiarrítmicos, anti-hipertensivos, analgésicos, anticonvulsivante, sedativos e opióides (Moraes et al., 2020). Portanto, avaliar os riscos inerentes a combinação de medicamentos, utilização de mecanismos de busca e identificação de IM por meio da prescrição eletrônica e envolver a equipe multiprofissional auxiliam na redução de eventos adversos a medicamentos.

Ainda Mazzola et al., (2011), no que se refere aos medicamentos mais utilizados em UTI, estão associados a protocolos como: sedação, analgesia, profilaxia de úlcera de estresse, profilaxia de tromboembolismo e controle da glicemia. Durante a análise dos dados, foram observados 10 potenciais interações medicamentosas teóricas (PIMT) mais comuns, entre elas, a dipirona obteve maior percentual de interação. Em relação à gravidade da interação, observou-se alta prevalência de PIMT moderadas, quando resulta em exacerbação do quadro clínico e/ou requer mudanças na terapia medicamentosa, e maiores, quando torna-se crônica e/ou requer intervenção médica para minimizar ou prevenir reações adversas graves. Diante 
das prescrições analisadas $88,2 \%$ apresentaram pelo menos uma PIMT, o que torna indubitável a necessidade de avaliação e monitoramento das prescrições.

Outros aspectos que levam a ocorrência de erros, segundo os estudos de Siman et al., (2021), são: prescrições médicas manual e ilegível, sobrecarga de trabalho, falta de atenção e capacitação profissional, grande número de pessoas circulando e interrupções no trabalho. Além disso, foram identificadas também falhas em todas as etapas de administração dos medicamentos, que implica no uso inadequado, podendo ou não lesar o paciente. Porém, é válido destacar, que todos esses aspectos são evitáveis e podem ser facilmente resolvidos se instituídas medidas de segurança corretas e eficientes.

Segundo Escrivá Gracia et al., (2019) os enfermeiros não possuem um nível de conhecimento necessário sobre os medicamentos que são mais utilizados e com os quais acontecem maior número de erros de medicação na UTI. As principais áreas de risco foram erros no intervalo de administração de antibióticos $(\mathrm{n}=8,15 \%)$; diluição, concentração e taxa de infusão de medicamentos de alto risco $(n=2,94 \%)$; e na administração de medicamentos por sonda nasogástrica $(n=11,16 \%)$. Nesse contexto, existem alguns fatores que podem estar relacionados a esses erros, como: o índice elevado de prescrição simultânea de medicamentos, o que pode acarretar em interações medicamentosas; e falha na comunicação das equipes, tornando um problema ainda maior.

Outro fator que deve ser avaliado com atenção no âmbito da UTI, refere-se a alta incidência de interações medicamentosas. A literatura aborda esse evento clínico quando existe a inferência nos efeitos de um fármaco na presença de outro fármaco, alimento, bebida ou algum agente químico ambiental (Storpirtis et al., 2013).

Em um estudo realizado por Yunes et al., (2011), em um hospital privado de Minas Gerais, observou-se que em 30\% das prescrições foram encontradas interações medicamentosas de alta gravidade (ex. enoxaparina e clopidogrel) já as interações de moderada gravidade corresponderam a 70\% (ex. sinvastatina e clopidogrel). Essas interações podem levar a deterioração do quadro clínico do paciente, trazendo a necessidade de terapia adicional, maior tempo de internação, aumento do custo hospitalar e transtorno desnecessário ao indivíduo. Neste sentido, ressalta-se a necessidade de conhecimento sobre interações medicamentosas, pois ao reconhecê-las precocemente é possível evitar terapias adicionais e obter êxito no tratamento.

Segundo Arboit et al., (2020) foi possível observar que alguns fatores contribuíram para a ocorrência de incidentes/interações, como: as rotinas de trabalho, a alta complexidade do quadro clínico do paciente, o cuidado fragmentado, estrutura física, carga de trabalho exaustiva, baixo índice de reconhecimento profissional e falta de atenção profissional. Entretanto, a conferência das prescrições e dos rótulos dos medicamentos, a dupla checagem, a passagem de plantão adequada, a identificação do paciente e a implementação dos "certos" da medicação são indicados como fatores humanos que acabam minimizando a ocorrência destes. Ressalta-se, ainda, que a conferência das prescrições médicas e dos rótulos das medicações, a não administração de medicamentos na ausência de conhecimento e/ou dúvidas, a identificação correta dos pacientes e dos medicamentos foram aludidos como estratégias para a prevenção da ocorrência de eventos adversos relacionados à medicação.

No que tange a segurança do paciente, a prática profissional é permeada por situações que podem desencadear um processo de riscos ao paciente. Dentre as atividades do enfermeiro, no âmbito da segurança do paciente destacam-se principalmente o estabelecimento de protocolos, a criação de barreiras de risco, a identificação do paciente, a dose unitária de medicamentos e a dupla checagem. Ressaltam-se também recomendações que envolvem a atuação profissional tais como: dedicação no trabalho, humanização, respeito e empatia (De Mello \& Barbosa, 2013).

A preocupação com a segurança do paciente vem sendo desenvolvida de forma crescente entre os pesquisadores de todo o mundo. Com isso, os medicamentos de alta vigilância ou "potencialmente perigosos" (MPP) têm tomado um lugar importante no que se refere às medidas de prevenção de erros (Reis et al., 2018). Sendo assim, o cuidado de enfermagem torna-se fundamental no processo do cuidar associado à prática segura de medicamentos. 
$\mathrm{Na}$ área da saúde, os erros estão associados à figura de "maus profissionais", problemas pessoais, aos erros de comportamento e/ou erros intelectuais. Outrora, os modelos de gestão foram pautados na cultura de punição, não sendo essa a forma ideal de trabalho. Dessa forma, a Portaria no 529/2013 do Ministério da Saúde, em conjunto com a Resolução da Diretoria Colegiada $n^{\circ} 36 / 2013$, veio consolidar a necessidade de mudança nos conceitos, substituindo a cultura de punição pela cultura de segurança do paciente em serviços de saúde.

\subsection{O cuidado farmacêutico na assistência a pacientes críticos}

O farmacêutico, historicamente, é visto como o profissional do medicamento, com foco apenas na gestão de farmácias hospitalares. Nas últimas décadas, esse paradigma vem se modificando e após a publicação de resoluções que dispõem sobre atribuições clínicas desse profissional, a sua inserção na equipe multiprofissional está sendo ampliada e paralelamente, permite promover o uso racional de medicamentos (Aparecida Maioli et al., 2018; Cedraz \& Junior, 2014).

O farmacêutico clínico pode contribuir significativamente na segurança do paciente, principalmente, aqueles sob cuidados intensivos, pois devido à complexidade das doenças, o emprego de medicamentos de alto risco e a regimes medicamentosos críticos aumenta a ocorrência de eventos adversos a medicamentos (Pilau et al., 2014).

As ações clínicas realizadas pelo farmacêutico baseiam-se, principalmente, na avaliação de prescrições médicas. A revisão da farmacoterapia para cada paciente, consiste na análise de dados antropométricos, história clínica, indicação terapêutica, aprazamento e via de administração, duplicidade de medicamentos e a presença de interações medicamentosas, sendo uma atividade de extrema importância para otimização dos resultados clínicos e segurança do paciente (Gomes \& Reis, 2003; Ribeiro et al., 2015). Além disso, ações de acompanhamento farmacoterapêutico, disponibilidade de medicamentos prescritos, conciliação de medicamentos, monitoramento das concentrações plasmáticas da droga e participação em visitas multidisciplinares também são realizadas (Milani et al., 2018).

Nessa perspectiva, a implantação de serviços clínicos farmacêuticos (SCF) vem demonstrando uma ferramenta capaz de melhorar a qualidade assistencial (Milani et al., 2018). A partir da revisão de estudos realizados é possível reconhecer pontos críticos no processo de cuidado e traçar estratégias para evitar ou minimizar danos decorrentes da utilização de medicamentos.

Reforçando essa ideia, o trabalho realizado por Okumura et al., (2016), o qual avaliou a implementação dos serviços clínicos em Unidades de Terapia Intensiva Pediátrica (UTIP) de um hospital comunitário no Sul do Brasil, detectou 141 PRMs nas prescrições analisadas, dentre eles os problemas mais prevalentes foram: incompatibilidade de soluções intravenosas e doses inadequadas, na qual demandaram dos farmacêuticos clínicos sugestões de intervenção a fim de prevenir erros evitáveis. Os autores reforçam, que o sucesso da implantação desse tipo de serviço na UTI é baseado em dois aspectos: o apoio institucional e a comunicação entre farmacêutico e o diretor clínico, a equipe de enfermagem e também, a Comissão de Controle de Infecção Hospitalar.

Já um estudo descritivo e retrospectivo realizado por residentes do Programa de Atenção ao Paciente Crítico, em um hospital universitário em Campo Grande - MS, conseguiu traçar o perfil dos problemas farmacoterapêuticos (n=183), no qual foi constatado que os desvios mais recorrentes foram identificados na prescrição $(n=44,9 \%)$, na dispensação $(n=22,0 \%)$, na administração $(n=21,1 \%)$ e no monitoramento da concentração plasmática de medicamentos $(n=10,5 \%)$. Mediante a esses desvios, observou-se que as drogas normalmente envolvidas nos problemas foram: omeprazol, metoprolol, enoxaparina, ranitidina, vancomicina e hidrocortisona. Nesse sentido, os farmacêuticos residentes gerenciavam as incompatibilidades e as interações medicamentosas identificadas, propondo intervenções $(n=129)$ que, em geral, traziam alternativas terapêuticas em relação ao que foi prescrito anteriormente (Milani et al., 2018). 
Em outro estudo brasileiro, realizado em hospital universitário durante 3 anos, foi observado as atividades clínicas do farmacêutico no âmbito da equipe multiprofissional presente em uma UTI. Nessa pesquisa, foram registradas 843 recomendações farmacêuticas (RF), sendo as mais frequentes: o ajuste de dose; a suspensão da terapia; manejo de eventos adversos, entre eles o manejo de interações medicamentosas; e recomendações quanto à provisão de medicamentos, devido à indisponibilidade na instituição. Entre essas RF, os anti-infecciosos foram os que tiveram maior participação (n=52,7\%). Diante desse cenário, os autores reforçam a importância da ampliação do cuidado farmacêutico à beira do leito, pois isso pode refletir no aumento da segurança do paciente (Fideles et al., 2015).

Ao avaliar os dados apresentados por Li et al., (2020), a partir de estudo prospectivo conduzido em um hospital acadêmico em Pequim, na qual avaliou serviços farmacêuticos em uma unidade de terapia intensiva segundo um modelo de cuidado proposto pela equipe de farmácia. Como resultado, foram atendidos 354 pacientes, foram identificados durante consulta farmacêutica 427 PRM e foram realizadas 486 intervenções. Dentre os PRMs detectados aqueles mais prevalentes foram: eventos adversos a medicamentos, tratamento não efetivo, principalmente de antibióticos, e prescrição de drogas desnecessárias. As causas do insucesso terapêutico estavam relacionadas à escolha inadequada de drogas de acordo com as diretrizes, tratamento incompleto; já a ocorrência de eventos adversos a medicamentos estava associada ao emprego de altas doses e por período muito longo de uso. Assim, foi possível perceber a eficácia de utilização de um modelo sistematizado do cuidado farmacêutico e a interação desse profissional com a equipe multiprofissional contribui para segurança do paciente crítico.

Neste contexto, um trabalho realizado por Maioli e colaboradores (2018), relataram as vantagens de realizar o cuidado farmacêutico baseado em processos sistematizados e documentados que garantam maior efetividade da assistência prestada. No estudo, eles discutiram o "Fast Hug", um checklist composto por 7 itens essenciais durante a avaliação de prescrições de pacientes em cuidados intensivos, como ferramenta na identificação e prevenção de erros de medicação. Esses itens fazem referência: alimentação, analgesia, sedação, profilaxia do tromboembolismo venoso, cabeceira elevada e profilaxia da úlcera por estresse, os quais promovem impacto na assistência desses pacientes.

Ao avaliar a repercussão da revisão de medicamentos como instrumento para detectar e prevenir erros evitáveis encontrados em prescrições de pacientes em UTIs, foi observado que dos 162 pacientes participantes do estudo, foram identificados 220 problemas relacionados a medicamentos dos quais 68,64\% poderiam resultar em eventos adversos. A partir da detecção de PRMs e as intervenções propostas pelo farmacêutico à equipe multiprofissional houve redução da frequência de eventos adversos a medicamentos, aumentando a segurança do paciente neste ambiente (Martins et al., 2019).

Mediante os estudos selecionados, ficou evidente que o farmacêutico pode contribuir significativamente com o sucesso farmacoterapêutico e com a prevenção de erros evitáveis no ambiente da UTI. Adicionalmente, a atuação desse profissional produz respostas diretas na diminuição dos custos com medicamentos e indiretos com a redução de reações adversas a medicamentos (RAMs) (Pilau et al., 2014).

Entretanto, existem alguns desafios que precisam ser superados para efetiva participação desse profissional no cuidado direto do paciente. Embora algumas resoluções e recomendações que reafirmam a importância dos serviços clínicos farmacêuticos, na prática isso não acontece. Além disso, há uma lacuna de profissionais farmacêuticos capacitados e treinados para execução de serviços clínicos, assim como existe resistência por parte de alguns profissionais de saúde e gestores de hospitais (Li et al., 2020).

\section{Discussão}

Nos últimos anos, vem sendo discutido acerca da melhoria da qualidade dos serviços prestados a pacientes hospitalizados. Em 2013, o Ministério da Saúde definiu 6 metas fundamentais para salvaguardar o paciente, sendo que a 
terceira meta visa melhorar as práticas de utilização de medicamentos de Alta Vigilância, referentes à prescrição, uso e a administração. Além disso, ressalta a relevância de implantar e implementar estratégias que promovam a segurança do paciente, dos familiares e dos profissionais em diferentes áreas da atenção, com a articulação e integração dos processos de cuidar e organizativo dos serviços de saúde (Brasil, 2013).

Diante disso, um ponto primordial para traçar estratégias que viabilizem práticas efetivas de segurança é justamente a identificação dos fatores que levam a ocorrência de desvios na durante a utilização do medicamento.

De acordo com um estudo realizado no Irã, foram apontadas as causas para ocorrência de erros de medicação no ambiente de UTI sob a perspectiva de alguns profissionais da saúde como: médicos, enfermeiros e farmacêuticos clínicos. Os autores classificaram esses erros em quatro categorias: 1) baixa atenção dos profissionais de saúde à segurança dos medicamentos, na qual observa-se a existência de prescrições médicas ilegíveis e incompletas, administração de medicamentos inadequada e erros de rotulagem; 2) falta de comunicação e colaboração profissional que acaba impactando na assistência integral do paciente; 3 ) determinantes ambientais que propiciam a ocorrência de erros como: a sobrecarga de trabalho, estresse e fatores emocionais; 4) determinantes de gestão relacionados ao quantitativos insuficiente de profissionais (Farzi et al., 2017).

Ao analisar um estudo feito por Chapuis et al., (2019), foi possível observar algumas estratégias as quais foram elencadas pelo comitê multidisciplinar, com intuito de diminuir o número de eventos adversos em UTI. Dentre essas, destacam-se: o emprego de sistema de dispensação automatizada; uso de prescrição eletrônica; sistema de vigilância ativa por meio da elaboração de relatórios que são posteriormente discutidos; realização de atividades de treinamento; e principalmente, aproximação dos profissionais da saúde quanto a tomada de decisões sobre os processos de cuidado.

Em relação a inserção da prescrição eletrônica, ferramenta tecnológica que auxilia na identificação precoce de eventos adversos a medicamentos, um estudo realizado por Li e demais colaboradores (2020) concluíram que logo após a implantação do sistema de prescrição eletrônica, houve um aumento no número de erros de medicação detectados, devido à falta de treinamento e de familiaridade ao novo sistema, entretanto, após 2 anos esse número reduziu significativamente. Além disso, observaram ainda, que o número de erros de prescrição também sofreu diminuição, por eliminar o problema da falta de legibilidade das prescrições.

Em virtude do assunto abordado, percebe-se que a utilização de recursos tecnológicos, somada a maior interação entre os profissionais de saúde, em especial, enfermeiros e farmacêuticos, são necessárias para reduzir drasticamente os eventos adversos relacionados à utilização de medicamentos em áreas como a UTI. Ademais, as limitações identificadas neste trabalho, podem ser incluídas: tamanho da amostra, carência de pesquisas anteriores sobre o tema e divergência de terminologias, o que dificultou a comparação entre os estudos incluídos nesta revisão.

\section{Considerações Finais}

No contexto hospitalar e principalmente quando se fala em pacientes hospitalizados em UTI é impossível não pensar em uma conjuntura: enfermagem, farmácia e medicamentos. Tais elementos são essenciais para alcançar o objetivo principal, uma assistência segura com menor erro possível, seja em procedimentos ou em administração de medicamentos.

Foi observado que apesar de existirem protocolos, metas e treinamentos em prol de uma assistência segura, ainda ocorrem erros evitáveis frequentemente. Para tanto, ao longo dos anos, as organizações de saúde vêm desenvolvendo estratégias e estabelecendo metas que promovam a redução de problemas relacionados a medicamentos e isso requer uma política de conscientização efetiva. Ainda assim, há necessidade constante de um olhar acurado, vigilante, humanista e social, para que paciente, família e profissionais sejam agentes coparticipantes do plano terapêutico, juntamente às capacitações e o fortalecimento das relações entre profissionais, para corroborar com a minimização de erros. Para tanto, faz-se necessária a 
integração e a articulação de enfermeiros e farmacêuticos para que a farmacoterapia seja efetiva, segura e assertiva, mediante a complexidade projetada na prática.

Sugerem-se mais pesquisas sobre a temática, visto a relevância do tema, para melhoria da assistência voltada ao uso de medicamentos em pacientes críticos, em especial as classes de antimicrobianos e medicamentos de alta vigilância. Propõese também novas pesquisas sobre a interação da equipe multiprofissional. Outra abordagem que pode ser adotada é a utilização de indicadores para validação de protocolos, melhoria dos treinamentos e capacitação dos profissionais e posterior debriefing. Sendo assim, observa-se diariamente a importância destes profissionais para assegurar a qualidade da assistência a pacientes internados em UTI.

\section{Referências}

Aparecida Maioli, N., Fernanda dos Santos Ferrari, A., Domingos dos Santos, T., \& Cesar Barbosa Santos, H. (2018). Fast Hug: Uma Ferramenta Para Farmácia Clínica Na Atenção E Segurança Do Paciente Crítico. Colloquium Vitae, 10(2), 59-64. https://doi.org/10.5747/cv.2018.v10.n2.v233.

Arboit, É. L., Camponogara, S., Magnago, T. B. de S., Urbanetto, J. de S., Beck, C. L. C., \& Silva, L. A. A. da. (2020). Factors contributing to the incident occurrence of security related to drug use in intensive care. Revista de Pesquisa Cuidado é Fundamental Online, 12, 1030-1036. https://doi.org/10.9789/21755361.rpcfo.v12.7456.

Borges, M. D., Correia, D. M. S., Hipólto, R. L., Christovam, B. P., Santo, F. H. E., Debora, K. V., \& Batista, D. C. S. (2017). Pesquisa-ação com foco em cardiotônicos e interações para prática segura de enfermagem. Saude Coletiva, 49(09), 1644-1650.

Brasil, M. da S. (2013). Portaria n.529, de $1^{\circ}$ de abril de 2013. Institui o Programa Nacional de Segurança do Paciente (PNSP). In Diário Oficial da União. https://bvsms.saude.gov.br/bvs/saudelegis/gm/2013/prt0529_01_04_2013.html

Brasil. Ministério da Saúde. Agência Nacional de Vigilância Sanitária. Resolução da Diretoria Colegiada - RDC nº 36 , de 25 de julho de 2013. Institui ações para a segurança do paciente em serviços de saúde e dá outras providências. Diário Oficial da União, Brasília, DF, 26 jul. 2013. p. 32.

Cedraz, K. N., \& Junior, M. C. dos S. (2014). Identification and characterization of drug interactions in prescriptions of the intensive care unit of a public hospital in the city of Feira de Santana, BA. Rev Soc Bras Clin Med, 12(2), 124-130.

Chapuis, C., Chanoine, S., Colombet, L., Calvino-Gunther, S., Tournegros, C., Terzi, N., Bedouch, P., \& Schwebel, C. (2019). <p>Interprofessional safety reporting and review of adverse events and medication errors in critical care $</ \mathrm{p}>$. Therapeutics and Clinical Risk Management, Volume 15, 549-556. https://doi.org/10.2147/TCRM.S188185

De Mello, J. F., \& Barbosa, S. de F. F. (2013). Cultura de segurança do paciente em terapia intensiva: Recomendações da enfermagem. Texto e Contexto Enfermagem, 22(4), 1124-1133. https://doi.org/10.1590/S0104-07072013000400031

Escrivá Gracia, J., Brage Serrano, R., \& Fernández Garrido, J. (2019). Medication errors and drug knowledge gaps among critical-care nurses: A mixed multimethod study. BMC Health Services Research, 19(1), 1-9. https://doi.org/10.1186/s12913-019-4481-7

Farzi, S., Irajpour, A., Saghaei, M., \& Ravaghi, H. (2017). Causes of medication errors in intensive care units from the perspective of healthcare professionals. Journal of Research in Pharmacy Practice, 6(3), 158. https://doi.org/10.4103/jrpp.jrpp_17_47

Fideles, G. M. A., De Alcântara-Neto, J. M., Júnior, A. A. P., De Souza-Neto, P. J., Tonete, T. L., Da Silva, J. E. G., \& Neri, E. D. R. (2015). Pharmacist recommendations in an intensive care unit: Three-year clinical activities. Revista Brasileira de Terapia Intensiva, 27(2), 149-154. https://doi.org/10.5935/0103-507X.20150026

Gomes, M. J. V. de M., \& Reis, A. M. M. (2003). Ciências Farmacêuticas Uma Abordagem em Farmácia Hospitalar. Atheneu.

Guzzo, G. M., de Magalhães, A. M. M., de Moura, G. M. S. S., \& Wegner, W. (2018). Medication safety in neonatology: Nursing in the perspective of the ecological restorative approach. Texto e Contexto Enfermagem, 27(3), 1-10. https://doi.org/10.1590/0104-070720180004500016

Li, X. X., Zheng, S. Q., Gu, J. H., Huang, T., Liu, F., Ge, Q. G., Liu, B., Li, C., Yi, M., Qin, Y. F., Zhao, R. S., \& Shi, L. W. (2020). Drug-Related Problems Identified During Pharmacy Intervention and Consultation: Implementation of an Intensive Care Unit Pharmaceutical Care Model. Frontiers in Pharmacology, 11. https://doi.org/10.3389/fphar.2020.571906

Martins, R. R., Silva, L. T., \& Lopes, F. M. (2019). Impact of medication therapy management on pharmacotherapy safety in an intensive care unit. International Journal of Clinical Pharmacy, 41(1), 179-188. https://doi.org/10.1007/s11096-018-0763-0

Mazzola, P. G., Rodrigues, A. T., Da Cruz, A. A., De Marialva, M., Granja, S., Battaglini, S. C. M., Falcao, A. L. E., \& Moriel, P. (2011). Artigo Original Perfil E Manejo De Interações Drug Interactions In Icu Prescriptions. Universidade Estadual de Campinas - UNICAMP, 2, 15-19.

Milani, A. C., De Oliveira Araujo, E., \& Guimarães Polisel, C. (2018). Pharmacotherapeutic problems and pharmaceutical interventions in critical hospitalized patients. O Mundo Da Saúde, 42, 369-292. https://doi.org/10.15343/0104-7809.20184202369392

Okumura, L. M., da Silva, D. M., \& Comarella, L. (2016). Relation between safe use of medicines and Clinical Pharmacy Services at Pediatric Intensive Care Units. Revista Paulista de Pediatria (English Edition), 34(4), 397-402. https://doi.org/10.1016/j.rppede.2016.04.001 
Research, Society and Development, v. 10, n. 6, e38410615862, 2021

(CC BY 4.0) | ISSN 2525-3409 | DOI: http://dx.doi.org/10.33448/rsd-v10i6.15862

Pereira, A., Shitsuka, D., Parreira, F., \& Shitsuka, R. (2018). Metodologia da Pesquisa Científica. In Metodologia da Pesquisa Científica (1a ed.) https://repositorio.ufsm.br/bitstream/handle/1/15824/Lic_Computacao_Metodologia-Pesquisa-Cientifica.pdf?sequence=1. Acesso em: 28 março 2020.

Pilau, R., Hegele, V., \& Heineck, I. (2014). Atuação do Farmacêutico Clínico em Unidade de Terapia Intensiva Adulto: Uma Revisão da Literatura. Rev. Bras. Farm. Hosp. Serv. Saúde São Paulo, 5(1), 19-24.

Reis, G. A. X. dos, Hayakawa, L. Y., Murassaki, A. C. Y., Matsuda, L. M., Gabriel, C. S., \& Oliveira, M. L. F. de. (2017). Nurse Manager Perceptions of Patient Safety Strategy Implementation. Texto \& Contexto - Enfermagem, 26(2), 1-9. https://doi.org/10.1590/0104-07072017000340016

Reis, M. A. S. dos, Gabriel, C. S., Zanetti, A. C. B., Bernardes, A., Laus, A. M., \& Pereira, L. R. L. (2018). Medicamentos Potencialmente Perigosos: Identificação De Potentially Hazardous Drugs : Identification of Risks and Error Prevention Barriers in Intensive Care. Texto \& Contexto - Enfermagem, 27(2), $1-9$.

Ribeiro, V. F., Sapucaia, K. C. G., Aragão, L. A. O., Bispo, I. C. da S., Oliveira, V. F., \& Alves, B. L. (2015). Realização de intervenções farmacêuticas por meio de uma experiência em farmácia clínica. Rev. Bras. Farm. Hosp. Serv. Saúde, 6(4), 18-22.

Siman, A. G., Tavares, A. T. D. V. B., Amaro, M. O. F., \& Carvalho, C. A. de. (2021). Medication error: conceptions and behaviors of the nursing team members. Revista de Pesquisa Cuidado é Fundamental Online, 109-116. https://doi.org/10.9789/2175-5361.rpcfo.v13.7853

Storpirtis, S., Mori, A. L. P. M., Yochiy, A., Ribeiro, E., \& Porta, V. (2013). Farmácia Clínica e Atenção Farmacêutica (Atheneu (ed.)).

Yunes, L. P., Coelho, T. de A., \& De Almeida, S. M. (2011). Principais interações medicamentosas em pacientes da Uti-adulto de um hospital privado de Minas Gerais. R. Bras. Farm. Hosp. Serv. Saúde São Paulo, 2 n.3(61), 23-26. http://www.sbrafh.org.br/rbfhss/public/artigos/RBFHSSV2N3 artigo04.pdf 